\title{
MAPK cascades and major abiotic stresses
}

\author{
Khaled Moustafa ${ }^{*}$, Synan AbuQamar, Mohammad Jarrar, Abdul Jabbar Al-Rajab, and \\ Jocelyne Trémouillaux-Guiller
}

\begin{abstract}
Plants have evolved with complex signaling circuits that operate under multiple conditions and govern numerous cellular functions. Stress signaling in plant cells is a sophisticated network composed of interacting proteins organized into tiered cascades where the function of a molecule is dependent on the interaction and the activation of another. In a linear scheme, the receptors of cell surface sense the stimulus and convey the stress signal through specific pathways and downstream phosphorylation events controlled by Mitogen-Activated Protein (MAP) kinases and second messengers, leading to appropriate adaptive responses. The specificity of the pathway is guided by scaffolding proteins and docking domains inside the interacting partners with distinctive structures and functions. The flexibility and the finetuned organization of the signaling molecules drive the activated MAP kinases into the appropriate location and connection to control and integrate the information flow. Here, we overview recent findings of the involvement of MAP kinases in major abiotic stresses (drought, cold and temperature fluctuations) and we shed the light on the complexity and the specificity of MAP kinase signaling modules.
\end{abstract}

Keywords: MAP kinases, mitogen activated protein, environmental abiotic stresses, plant adaptation.

\section{Abbreviations:}

MAP: Mitogen Activated Protein

MAPK: Mitogen Activated Protein Kinase

MAP2K, MEK, MKK, or MAPKK: MAPK kinase

MAP3K, MEKK MKKK or: MAPK kinase kinase

MAP4K, MKKK or MEKKK: MAPK kinase kinase kinase.

S/T: Serine/Threonine, T(E/D)Y: Threonine(Glutamate/Aspartate)Tyrosine 


\section{Introduction}

Drought, salinity, and temperature shifts are major abiotic environmental stresses that affect civilizations since the dawn of agriculture with recurring waves of different intensities and amplitude. MAP kinase cascades convey stress signals from receptors to specific effectors to regulate gene expression, cell activities and protein functions in various developmental and adaptive processes. The achievement of sequencing of some plant genomes reveal that MAP kinases are tiered into four gene family classes. Arabidopsis genome for example encodes for 10 MAP4Ks, 60 MAP3Ks, 10 MKKs, and 20 MPKs genes for which a new nomenclature and group classification was established (Ichimura, et al. 2002). Due to the lack of information of the MAP4Ks, MAP kinase signaling modules are typically described to be composed of three proteins, starting by a MAP kinase kinase kinase (known also as MAP3K, MEKK or MAPKKK), which activates a MAP kinase kinase (MAPKK, MAP2K or MKK), which, in turn, activates a MAP kinase (MAPK, also called MPK). The activation of these MAP kinases is a sequential process through the phosphorylation of some conserved residues within an activation loop in their kinase domains. Following an internal or external signal, a MAP3K protein phosphorylates a MAP2K in two serine/threonine residues, in the consensus motif $S / T x x x x x S / T$, which in turn activates a MAPK by phosphorylation on threonine and tyrosine residues in $T(E / D) Y$ conserved motif. MAP kinases, with phosphatases, function as On/Off signal switchers to regulate the activity of many downstream targets such as cytoskeletal proteins in the cytosol or transcription factors in the nucleus to control cell signaling in plant adaptation to environmental oscillations.

Although environmental factors are natural components of the ecosystem, hence hardly unavoidable hazards, sub- and supra optimal ranges of these factors disturb plant growth and reduce plant productivity by up to $50 \%$ (Bray, et al. 2000). To maintain plant productivity and sustain ecology under environmental harsh conditions, the development of new crop varieties is of great importance to secure food availability and quality under exacerbate climate change. The first step towards such an objective is to understand the molecular and genetic bases of plant interactions with its adverse environmental conditions. The integration of genetic and omics approaches such as genomics, transcriptomics, proteomics, and metabolomics have opened wide avenues towards the dissection of the adaptation mechanisms in several living organisms. Deeper understanding of such mechanisms helps the bioengineering of new adaptable plant cultivars in a relatively short time compared to classical breeding approaches. Here, we summarize recent findings in the involvement of MAP kinases signaling pathways in response to the most devastating abiotic stresses worldwide; drought, salt and extreme temperatures. The complexity of MAP kinase modules are also debated in the light of new findings.

\section{MAP kinase complexity in environmental stresses' signaling}


Unlike the interaction with a biotic stress, which is mostly provoked by one living factor, plant interaction with abiotic stresses, is multivariable complex trait. In natural ecosystem, one type of abiotic stresses comes rarely alone. Drought, salt and temperatures are closely related with overlapping physiological effects (Krasensky and Jonak 2012). High temperatures for example frequently come with drought and water deficits, aggravating the effects of salinity and osmotic shifts on plant cells (Bita and Gerats 2013) (Xiong, et al. 1999). Salinity is also a common feature in arid and semiarid lands where plants are convicted to tolerate osmotic stress caused by both salinity and low water potential (Rengasamy 2006). As a result, a complex signaling network should operate and activate relevant signaling pathways to allow plants survival.

Plant survival under multiple and simultaneous environmental conditions implies complex processes of signal reception, transmission, amplifications and interaction. The transmission of information in biological systems is rarely one way direction. Signal transmission is rather a complex crosstalk activity involving different molecules at different levels of organization. The large number of MAP kinases and stress responsive genes outlines this complexity and suggests how stressed plants struggle to adapt to diverse and adverse conditions.

Signal transmission complexity via MAP kinases is further layered by the fact that MAP kinases are multi-tiered gene families recruited for multiple functions such as development, immune defense system, hormones signaling and responses to biotic and abiotic environmental stresses. MAP kinase signaling is also convoluted by opposite regulation patterns under the same or different environmental stresses. In fact, while many MAP kinases are described to be positively regulated under abiotic stresses, some MAP kinases play negative roles in stress tolerance. For example, overexpression of the MAP kinases,

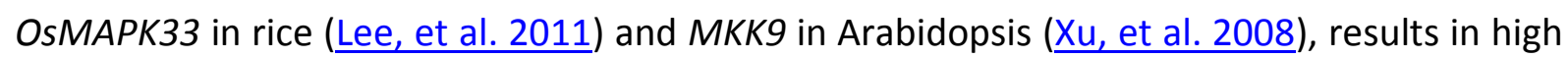
sensitivity of rice and Arabidopsis, respectively, to salt stress. Moreover, the inactivation of the Arabidopsis MKK9 by the insertion of transferred DNA (T-DNA) generates salt insensitive phenotypes that germinate at up to $150 \mathrm{mM} \mathrm{NaCl}$, with rapid induction of $R D 22$ and $R D 29$ stress genes (Alzwiy and Morris 2007). A mapkkk knockout mutant of the Arabidopsis MAPKKK20 (MAP kinase kinase kinase) presents an improved salt tolerance, particularly in the later developmental stages (Gao and Xiang 2008). Additionally, the Arabidopsis double mutant $m p k 9 / m p k 12$ loses water by transpiration less than the wild type (Jammes, et al. 2009).

The dichotomy of positive/negative regulation of MAP kinases in stress responses adds an extra layer of MAP kinases complexity and specificity that need to be elucidated. Due to a large number of homologous MAP kinases in plants, MAP kinases specificity is a tricky question that may lead to a possible redundancy in diverse biological processes. One powerful method to elucidate this question is to systematically silent MAP kinases, and then characterizing the resulting phenotypes. This should provide evidence answers to the redundancy or complementarity issue. 
The control of a plethora of cellular processes by MAP kinases implies fine tuning specificity mechanisms that are associated with MAP kinase activation and function. Specificity mechanisms have been extensively studied in yeast and mammals, and several proteins have been identified as scaffolding molecules that assemble different MAP kinases into one specific signaling pathway. Looking for analogous scaffolding proteins in plants revealed the presence of some MAP kinases that play dual roles as scaffold protein and kinase enzyme at the same time. For example, the MAPKKK OMTK1 (oxidative stress-activated MAP triple kinase 1), which belongs to the alfalfa MAPKKK family, plays a role as an adapter and a phosphate transporter at the same time (Nakagami, et al. 2004). Other studies report that MAP kinases would act as structural adaptors and enzymatic activators in yeast (Edmunds and Mahadevan 2004). The presence of docking domains that form dovetail joints between compatible components in a given signaling pathway is another mechanism of MAP kinase specificity (Tanoue, et al. 2000). The spatial localization of MAP kinases may also increase target specificity with the advantage that co-localization of interacting partners would save cells an important amount of energy and reduces physical and functional effects of interfering molecules at long distance courses.

Among the other unanswered questions in MAP kinase signaling is the identification of MAP kinase substrates in each physiological or biochemical condition. In a highthroughput phosphorylation approach, 48 potential substrates of MPK3 and 39 of MPK6 were identified (Feilner, et al. 2005). However, in vivo characterization will be required to confirm the potential interaction between these MAP kinases and their corresponding partners.

\section{MAPKs and drought}

Drought is one of the major, if not the most, detrimental abiotic stresses worldwide. Drought has devastating impacts not only on plant metabolism and productivity, but also at socioeconomic levels with extended damages. A number of transcriptional and biochemical studies have reported the involvement of MAP kinases in drought responses in many grassy and woody plants. In Arabidopsis for example, it was shown that the MAP kinase MPK6 is required to enhance plant tolerance to dehydration via the regulation of RNA decapping

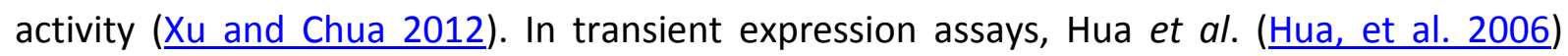
found that constitutively active forms of Arabidopsis MPK and MKK members are able to activate the promoter of $R D 29$, a dehydration responsive gene, suggesting that MAP kinase cascades are involved in drought signaling. The analysis of the transcriptional regulation of 44 MAP kinases under different abiotic stresses demonstrated that Arabidopsis MPK2, MPK3, MPK4, MPK5, MPK12 and MAPKKK4 are induced by water stress (Moustafa, et al. 2008). 
In rice (Oryza sativa), Ning et al. (Ning, et al. 2010) have identified a Raf like MAPKKK protein, named DSM1 (drought sensitive mutant1) that functions as a potential scavenger of the reactive oxygen species (ROS), increasing plant tolerance to dehydration under water stress. Drought tolerance in rice is also enhanced by overexpression of the MAP kinase OSMAPK5, an ortholog of the Arabidopsis MPK3 (Xiong and Yang 2003). A rapid induction of another rice MAP kinase, OSMSRMK2, was observed within $15 \mathrm{~min}$ in response to drought and increased levels of salt (Agrawal, et al. 2002), suggesting functional roles of the OsMSRMK2 in water stress signaling. Moreover, five of the ten members of the rice MAPKK family have been reported as transcriptionally regulated by drought (Kumar, et al. 2008).

At protein level, kinase activity of the alfalfa MAP kinase MMK4 is inducible by drought, though no correlation between protein activity and transcript levels was observed (Jonak, et al. 1996). This suggests that posttranslational regulation mechanisms may exist to modulate MAP kinase activity under stress conditions. In maize, a new MAP kinase, ZmMPK3, was also identified as a drought responsive gene (Wang, et al. 2010), and the genetic transformation with the tobacco MAPKKK NPK1 enhances the maize tolerance to drought (Shou, et al. 2004).

To improve the quality and adaptability of cotton (Gossypium spp.), extensive research is undertaken in many research centers. Recently, Zhang et al. (Zhang, et al. 2011) have identified GhMPK2 as a cotton MAP kinase that functions in reducing water loss and adjusting osmotic pressure under drought conditions. Another new cotton MAP kinase, called GhMPK16, belonging to the MAPK group D, was also identified as a drought responsive gene (Shi, et al. 2011). Subcellular localization analysis revealed that both GhMPK2 and GhMPK16 translocate into the nucleus, suggesting that both kinases are potential activators of transcription factors that function under drought conditions.

In woody and higher plants, MAP kinases also respondent to drought stress. In apple (Malus spp.), the MAP kinase MaMPK is upregulated at transcriptional and protein levels in three apple species with strong activity in the drought-tolerant species Malus sieversii, indicating that MaMPK could be associated with the natural drought tolerance trait in apple. (Peng, et al. 2006)

\section{MAPKs and osmotic stress}

Plant responses to salt stress involve different adaptive mechanisms such as ion exclusion and sequestration into the vacuole, particularly $\mathrm{Na}^{+}$and $\mathrm{Cl}^{-}$to reduce their cytotoxicity. The synthesis of protective proteins, such as LEA (late embryogenesis abundant) proteins and chaperones (e.g., heat shock proteins) is also accelerated to buffer the negative effects of the toxic ions. As signal transducers, MAP kinases convey osmotic stress signals to appropriate effectors and contribute to the adaptation of plant cells to the high salt concentrations. Research data from several studies support this assumption and show that MAP kinases are regulated by osmotic and salt stresses at both transcriptional and protein 
activity levels. Recently, Hoang et al. (Hoang, et al. 2012) have reported that the MAP kinase MPK6 activates, and interacts with, MYB41 and results in enhanced salt tolerance in Arabidopsis. In addition, the regulation of MPK6 by the phosphatidic acid (PA) under salt stress suggests that MPK6 and PA may function in the same signaling pathway in response to salt stress (Yu, et al. 2010).

A potential MAP kinase signaling pathway mediating salt stress responses, composed of MEKK1, MKK2 and the interchangeable MPK4/MPK6 genes was previously identified in Arabidopsis (Teige, et al. 2004). Transgenic plants overexpressing MKK2 exhibit constitutive upregulation of stress marker genes and show enhanced salt tolerance. The expression of different active forms of the MKKs and MPKs gene family members results in the activation of $R D 29 A$ and $R D 29 B$ gene promoters ( be upstream activators of the stress marker genes under salinity conditions. Additionally, five MPKs (MPK9, MPK10, MPK11, MPK17 and MPK18), two MKKs (MKK7 and MKK9) and four MEKKs (MEKK3, MEKK5, MEKK6, and MEKK7) were induced by $200 \mathrm{mM}$ sodium chloride ( $\mathrm{NaCl}$ ( (Moustafa, et al. 2008). Arabidopsis transgenic plants that overexpress the maize MAP kinase ZmSIMK1 showed an enhanced tolerance to high salinity stress (Gu, et al. 2010).

Recently, a new MAP kinase kinase kinase, called MKKK20, has been identified as a potential regulator of osmotic stress in Arabidopsis through the activation of MPK6 (Kim, et al. 2012). The Arabidopsis mkkk20 mutant exhibits high rates of water loss and superoxide accumulation, while transgenic plants overexpressing MKKK20 exhibit an enhanced tolerance to osmotic stress provoked by $\mathrm{NaCl}$, mannitol or sorbitol, suggesting important roles of MAPKKK20 in osmotic adjustment and stress tolerance (Kim, et al. 2012). Previously, it has been reported that Arabidopsis MKK4 was induced at transcriptional level in Arabidopsis cells and seedlings, and it mediates the osmotic stress responses via the kinase MPK3 (Kim, et al. 2011). The induction by osmotic stress of the Arabidopsis MPK17 and its homolog in maize ZmMPK17 (Pan, et al. 2012), suggests that MPK17 and its ortholog could be good candidates for further functional characterizations and involvement in abiotic stress adaptation. Interestingly, under hypoosmotic stress, the Arabidopsis MPK20 presents an induction pattern similar to that of proline dehydrogenase (ProDH) (Moustafa, et al. 2008), suggesting that MPK20 and ProDH may interact with each other and be part of functional hypoosmotic stress-signaling pathway.

In alfalfa, the MKK member, SIMKK, functions as an upstream activator of the SIMK (a MAPK) under salt stress conditions (Kiegerl, et al. 2000). Osmotic stress also induces a rapid and transient activation of the salicylic acid-induced kinase (SIPK, an Arabidopsis MPK6 homolog in tobacco) in tobacco cells within 5 to10 min (Mikolajczyk, et al. 2000).

In maize, Kong et al. have recently isolated a novel MAP kinase kinase, named ZmMKK4, whose expression is modulated by salinity stress. The overexpression of $Z m M K K 4$ in tobacco plants results in enhanced osmotic regulation (Kong, et al. 2011). 
In rice, several studies demonstrate that MAP kinases are implicated in salt stress tolerance. For example, the MAP kinases OsMPK44 (Jeong, et al. 2006), OsMSRMK3 (Agrawal, et al. $\underline{2003}$ ), OsMSRMK2 (Agrawal, et al. 2002), OsEDR1 (Kim, et al. 2003), OsMAPK5 (Xiong and Yang 2003), and OsMAPK4 ( $\mathrm{Fu}$, et al. 2002) have been identified as salt stress responsive genes. Moreover, rice transgenic lines overexpressing OSMPK5 and OSMPK44 demonstrate an improved tolerance of salt (Jeong, et al. 2006; Xiong and Yang 2003). Similarly, transgenic cotton overexpressing GhMPK2 exhibit an enhanced osmotic adjustment and increased salt tolerance (Zhang, et al. 2011). Finally, it was shown that the MAP kinase, CSNMAPK, is induced in cucumber roots upon salt treatments and the tobacco transgenic seeds overexpressing CSNMPAK exhibit higher germination rates compared to the wild type ( $\underline{\mathrm{Xu}}$, et al. 2010), suggesting that CSNMPAK plays positive roles in plant adaptation to salt stress at early stages.

\section{MAPKs and temperature stresses}

Temperature is an essential environmental factor for plant growth, metabolism and productivity. Due to large amount of water in plant tissues, the behavior of cell water under different temperatures is a key factor for cell survival. Depending on species and developmental stages, plants can grow healthy in wide ranges of temperatures (Went 1953). Beyond these ranges, plants could suffer from severe temperature damages. Low temperatures can inhibit germination, growth and metabolic activities (Roberts 1988). Cold stress also provokes water deficit and disturbs the membrane integrity by the formation of icy crystals (Yamazaki, et al. 2009). High temperatures, on the other hand, provoke heat stress and accelerate the respiration (Abele, et al. 2002) and the photosynthetic rates (Pastenes and Horton 1996), leading to overproduction of reactive oxygen species that affect plant growth and productivity.

So far, no specific receptor has been identified in plants in response to temperature stresses. However, the plasma membrane itself could play the role of primary sensor of temperature fluctuations (Sangwan, et al. 2002). In fact, the structural modifications in plasma membrane caused by temperature shifts can trigger downstream specific signaling events. While low extreme temperatures increase the rigidification of the plasma membrane, high temperatures increase its fluidization. Such fluctuations alter the activity of the membrane-associated proteins such as ATPases, ions transporters, $\mathrm{H}^{+}$pumps and protein channels. Both changes (rigidification and fluidization) are perceived as primary stimuli signals that trigger fluxes of second messengers (e.g. $\mathrm{Ca}^{+2}$ ) to activate specific heat response. Heat responses include the production and activation of heat shock proteins (HSPs) (Vierling 1991); heat shock transcription factors (HSFs); cold shock domain containing proteins (CSDPs) (Karlson and Imai 2003); ROS scavengers; antioxidants; compatible solutes; calcium dependent protein kinases (CDPKs) and MAP kinases.

Although little is known about MAP kinase signaling in responses to heat in plant, specific MAP kinases are upregulated and/or activated by temperature stresses. The heat shock 
activated MAP kinase (HAMK) was identified as the first heat responsive MAP kinase in alfalfa cells (Sangwan and Dhindsa 2002). The activation of HAMK in tobacco cells was observed conjunctly with the accumulation of heat shock protein, HSP70, suggesting that HAMK may trigger a heat signaling cascade via HSP genes (Suri and Dhindsa 2008). The activation of HAMK also seems to be calcium dependent in tomato leaves and cells exposed to heat stress (Link, et al. 2002). Heat and calcium-dependent activation of MAP kinases is also observed for MPK6 in Arabidopsis to regulate the activity of vacuolar processing enzyme (VPE) (Li, et al. 2012). In a recent study (Kong, et al. 2012), it was shown that the transcript abundance of the tomato MAP kinase SIMAPK increases after heat treatment.

In rice, a part of potentially interacting MAP kinases cascade, composed of OsMKK6OsMPK3, has been recently identified in response to chilling (Xie, et al. 2012). In the same way, OsMEK1 (a putative rice MAP kinase kinase, isolated by subtractive screening of cDNA library) has been shown to interact with OsMAP1 at $12^{\circ} \mathrm{C}$, suggesting that both kinases, OsMEK1 and OsMAP1, may be involved in the temperature stress signaling ( $\underline{\text { Wen, et al. }}$ 2002). Another MAP kinase, called OsMAPK4, has also been identified in rice leaves and

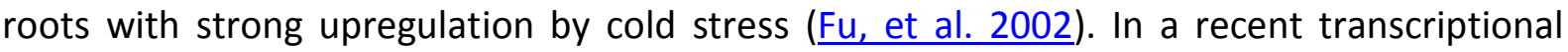
profiling study carried out on cassava, a tropical drought tolerant crop, an homologous of the Arabidopsis MPK4 was identified as one of the genes upregulated by cold stress $\left(7^{\circ} \mathrm{C}\right)$ (An, et al. 2012). Surprisingly, the mRNA transcripts of the rice OsMSRMK2 are regulated at both high $\left(37^{\circ} \mathrm{C}\right)$ and low-moderate $\left(12^{\circ} \mathrm{C}\right)$ temperatures (Agrawal, et al. 2002$)$. OsMKK4 and OsMKK6 are also strongly regulated by cold and heat treatments (Kumar, et al. 2008). Such contradictory results in opposite conditions could be explained by great functional plasticity of the MAP kinase signalling pathways.

In maize, a MPKK, ZmMKK4 (Kong, et al. 2011) and a MPK ZmMPK17 ( 2012) have been described to be inducible by cold stress. Both proteins are mainly located in the nucleus, suggesting that they regulate the activity of transcription factors functioning in plant adaptability to cold stress. This assumption may be supported by a high germination rate of tobacco seeds that overexpress $Z m M P K 17$ and accumulate high levels of proline. Five other MAP kinases were upregulated in maize roots at low temperatures ( $\underline{\mathrm{Wu}}$, et al. 2011). In cotton, the transcript level of the MAP kinase, GhMAPK, increased markedly in plant seedlings subjected to cold temperature $\left(4^{\circ} \mathrm{C}\right.$ ) (Wang, et al. 2007), suggesting that MAP kinases may play protective roles against cold effect on root cells.

The previously mentioned MAPK cascade (composed of MEKK1, MKK2, and MPK4/MPK6), which functions in salt stress signaling in Arabidopsis, is also functional in cold stress pathway (Teige, et al. 2004). The mkk2 mutant presents a freezing sensitive phenotype with no observable activity neither for MPK4 nor MPK6, suggesting that MKK2 is an upstream activator of MPK4 and MPK6 in cold signaling. This finding is consistent with previous studies showing that cold stress activates MEKK1, MPK3, MPK4 and MPK6 (Mizoguchi, et al. 1996) (Ichimura, et al. 2000). 
Finally, the MAP kinase kinase SbMAPKK isolated from salt tolerant species, Salicornia brachiate, is induced by many abiotic stresses with maximum expression levels at cold treatment (Agarwal, et al. 2010), suggesting that cold and salt stresses may have common kinase components that allow plants to cope with these harsh environmental conditions. It is also worthy to note that some MAP kinases are involved in the physiological recovery after the exposure to cold stress. For example, the transcript levels of the maize MAP kinase ZmMPK5 increase after a temperature shift from $5^{\circ}$ to $25^{\circ} \mathrm{C}$ (Berberich, et al. 1999). Table (1), summarizes a list of MAP kinase genes involved in the responses to multiple abiotic stresses in different plant species, based on changes in transcriptional regulation and/or enzymatic activities.

\section{Perspective and concluding remarks}

Several MAP kinases have been identified in response to multiple environmental stresses. MAP kinases serve as signal carriers and scaffolding proteins, linking complex arrays of interacting molecules in developmental and adaptive responses. In the forthcoming years, there will be an urgent need to identify MAP kinase substrate and develop new biotechnological approaches that accelerate the breeding of new plant species that should be well-adapted to environmental fluctuations to secure food supply for growing population. Mainly, it would be essential to identify and quantify interacting proteins that act coordinately under multiple environmental stresses. With their mode of action in a sequential way (MAPKKK-MAPKK-MAPK), MAP kinases offer an interesting potential to be assembled into one functional "MAPK transgenic circuits" to be delivered into target cells as a 'gene kit' or a 'gene circuit'. To accomplish such an approach, research is needed to determine the specific interacting MAP kinases and their targets and to define where and when these interacting partners may provide new characteristics of interest. Based on gene expression data and information presented in Table 1, an inferred MAP kinase cascade can be designed (figure 1) and tested in future research for the assessment of plant tolerance to abiotic stresses. The identification of MAPK targets would also help in the construction of complete functional proteins circuit from receptor to final target genes. Transgenic circuit directing protein-protein interactions hold the promise of consequential effects, and may provide a better transformation efficacy than the conventional genetic transformation based on only one single gene. Rational designing of transgenic circuit and taking into account the specific molecules that are most likely to interact with each other, is an important criterion for the success of such an approach. Wiring interacting molecules in a transgenic circuit and delivering it into plant cell would maintain the functional equilibrium of the interacting partners and result in efficient and synergetic adaptive responses.

Much also can be learned about MAP kinase functions, interaction and complexity network from experimental and theoretical studies as well, such as systems biology and in silico predictions. However, it is indispensable to investigate MAP kinase functions and interacting partners in vivo at whole plant levels. In vivo molecular imaging under stress conditions may help identifying interacting molecules at both qualitative and quantitative 


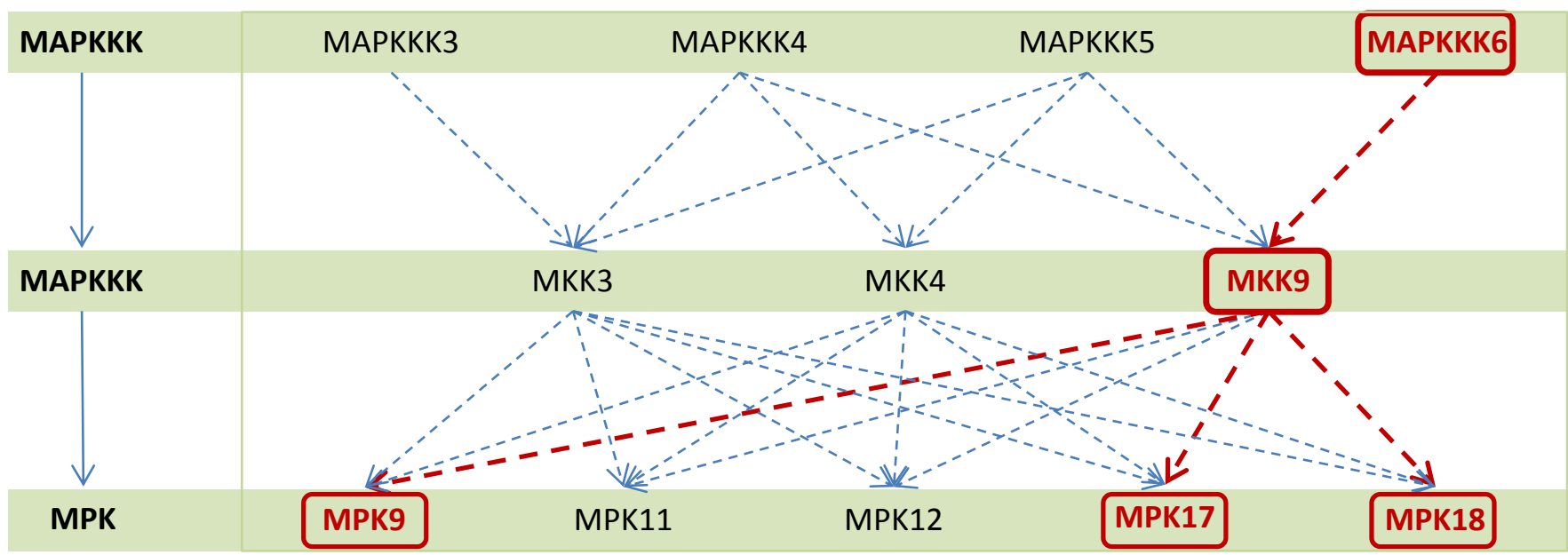

Figure 1. Hypothetic MAP kinase cascade inferred from gene expression data and co-regulation paterns.

Based on gene expression profiling, gene co-regulation and induction patterns by major abiotic stresses (drought, salt and temperatures) (Table 1), a hypothetic MAP kinase cascade (in red) could be investigated for plant tolerance to abiotic stresses :

\section{MAPKKK6 $\rightarrow$ MKK9 $\rightarrow$ MPK9/MPK17/MPK18}

Dotted arrows suggest potential interactions between MAP kinases in abiotic stress responses. 
levels. Such analysis, accomplished by the generation of MAP kinase mutants, will aid the determination of the precise signaling inputs and outputs involved in the organization and the regulation of MAP kinase cascades under multiple environmental abiotic stress conditions.

Conflict of interest: None.

\section{References}

Abele D, Heise K, Portner HO, Puntarulo S (2002) Temperature-dependence of mitochondrial function and production of reactive oxygen species in the intertidal mud clam Mya arenaria. The Journal of experimental biology 205:1831-1841

Agarwal PK, Gupta K, Jha B (2010) Molecular characterization of the Salicornia brachiata SbMAPKK gene and its expression by abiotic stress. Molecular biology reports 37:981-986

Agrawal GK, Agrawal SK, Shibato J, Iwahashi H, Rakwal R (2003) Novel rice MAP kinases OsMSRMK3 and OsWJUMK1 involved in encountering diverse environmental stresses and developmental regulation. Biochemical and biophysical research communications 300:775-783

Agrawal GK, Rakwal R, Iwahashi H (2002) Isolation of novel rice (Oryza sativa L.) multiple stress responsive MAP kinase gene, OsMSRMK2, whose mRNA accumulates rapidly in response to environmental cues. Biochemical and biophysical research communications 294:1009-1016

Alzwiy IA, Morris PC (2007) A mutation in the Arabidopsis MAP kinase kinase 9 gene results in enhanced seedling stress tolerance. Plant Science 173:302-308

An D, Yang J, Zhang P (2012) Transcriptome profiling of low temperature-treated cassava apical shoots showed dynamic responses of tropical plant to cold stress. BMC genomics 13:64

Berberich T, Sano H, Kusano T (1999) Involvement of a MAP kinase, ZmMPK5, in senescence and recovery from low-temperature stress in maize. Molecular \& general genetics : MGG 262:534-542

Bita CE, Gerats T (2013) Plant tolerance to high temperature in a changing environment: scientific fundamentals and production of heat stress-tolerant crops. Frontiers in plant science 4:273

Bray EA, J. B-S, E. W (2000) Responses to abiotic stress. In: Buchanan B, Grussem W, Jones R, Editors. Biochemistry and Molecular Biology of Plants. 1158-1203

Edmunds JW, Mahadevan LC (2004) MAP kinases as structural adaptors and enzymatic activators in transcription complexes. J Cell Sci 117:3715-3723

Feilner T, Hultschig C, Lee J, Meyer S, Immink RG, Koenig A, Possling A, Seitz H, Beveridge A, Scheel D, Cahill DJ, Lehrach H, Kreutzberger J, Kersten B (2005) High throughput identification of potential Arabidopsis mitogen-activated protein kinases substrates. Molecular \& cellular proteomics : MCP 4:1558-1568

Fu SF, Chou WC, Huang DD, Huang HJ (2002) Transcriptional regulation of a rice mitogen-activated protein kinase gene, OsMAPK4, in response to environmental stresses. Plant \& cell physiology 43:958-963 
Gao L, Xiang CB (2008) The genetic locus At1g73660 encodes a putative MAPKKK and negatively regulates salt tolerance in Arabidopsis. Plant Mol Biol 67:125-134

Gu L, Liu Y, Zong X, Liu L, Li DP, Li DQ (2010) Overexpression of maize mitogen-activated protein kinase gene, ZmSIMK1 in Arabidopsis increases tolerance to salt stress. Molecular biology reports 37:4067-4073

Hoang MHT, Nguyen XC, Lee K, Kwon YS, Pham HTT, Park HC, Yun D-J, Lim CO, Chung WS (2012) Phosphorylation by AtMPK6 is required for the biological function of AtMYB41 in Arabidopsis. Biochemical and biophysical research communications 422:181-186

Hua ZM, Yang X, Fromm ME (2006) Activation of the $\mathrm{NaCl}$ - and drought-induced RD29A and RD29B promoters by constitutively active Arabidopsis MAPKK or MAPK proteins. Plant, cell \& environment 29:1761-1770

Ichimura K, Mizoguchi T, Yoshida R, Yuasa T, Shinozaki K (2000) Various abiotic stresses rapidly activate Arabidopsis MAP kinases ATMPK4 and ATMPK6. The Plant journal : for cell and molecular biology 24:655-665

Ichimura K, Shinozaki K, Tena G, Sheen J, Henry Y, Champion A, Kreis M, Zhang S, Hirt H, Wilson C, Heberle-Bors E, Ellis BE, Morris PC, Innes RW, Ecker JR, Scheel D, Klessig DF, Machida Y, Mundy J, Ohashi Y, Walker JC (2002) Mitogen-activated protein kinase cascades in plants: a new nomenclature. Trends in plant science 7:301-308

Jammes F, Song C, Shin D, Munemasa S, Takeda K, Gu D, Cho D, Lee S, Giordo R, Sritubtim S, Leonhardt N, Ellis BE, Murata Y, Kwak JM (2009) MAP kinases MPK9 and MPK12 are preferentially expressed in guard cells and positively regulate ROS-mediated ABA signaling. Proceedings of the National Academy of Sciences of the United States of America 106:20520-20525

Jeong M-J, Lee S-K, Kim B-G, Kwon T-R, Cho W-S, Park Y-T, Lee J-O, Kwon H-B, Byun M-O, Park S-C (2006) A rice (Oryza sativa L.) MAP kinase gene, OsMAPK44, is involved in response to abiotic stresses. Plant Cell, Tissue and Organ Culture 85:151-160

Jonak C, Kiegerl S, Ligterink W, Barker PJ, Huskisson NS, Hirt H (1996) Stress signaling in plants: a mitogen-activated protein kinase pathway is activated by cold and drought. Proceedings of the National Academy of Sciences of the United States of America 93:11274-11279

Karlson D, Imai R (2003) Conservation of the cold shock domain protein family in plants. Plant physiology 131:12-15

Kiegerl S, Cardinale F, Siligan C, Gross A, Baudouin E, Liwosz A, Eklof S, Till S, Bogre L, Hirt H, Meskiene I (2000) SIMKK, a mitogen-activated protein kinase (MAPK) kinase, is a specific activator of the salt stress-induced MAPK, SIMK. The Plant cell 12:2247-2258

Kim J-M, Woo D-H, Kim S-H, Lee S-Y, Park H-Y, Seok H-Y, Chung W, Moon Y-H (2012) Arabidopsis MKKK20 is involved in osmotic stress response via regulation of MPK6 activity. Plant cell reports 31:217-224

Kim JA, Agrawal GK, Rakwal R, Han KS, Kim KN, Yun CH, Heu S, Park SY, Lee YH, Jwa NS (2003) Molecular cloning and mRNA expression analysis of a novel rice (Oryzasativa L.) MAPK kinase kinase, 
OsEDR1, an ortholog of Arabidopsis AtEDR1, reveal its role in defense/stress signalling pathways and development. Biochemical and biophysical research communications 300:868-876

Kim SH, Woo DH, Kim JM, Lee SY, Chung WS, Moon YH (2011) Arabidopsis MKK4 mediates osmoticstress response via its regulation of MPK3 activity. Biochemical and biophysical research communications 412:150-154

Kong F, Wang J, Cheng L, Liu S, Wu J, Peng Z, Lu G (2012) Genome-wide analysis of the mitogenactivated protein kinase gene family in Solanum lycopersicum. Gene 499:108-120

Kong X, Pan J, Zhang M, Xing X, Zhou Y, Liu Y, Li D (2011) ZmMKK4, a novel group C mitogen-activated protein kinase kinase in maize (Zea mays), confers salt and cold tolerance in transgenic Arabidopsis. Plant, cell \& environment 34:1291-1303

Kong X, Sun L, Zhou Y, Zhang M, Liu Y, Pan J, Li D (2011) ZmMKK4 regulates osmotic stress through reactive oxygen species scavenging in transgenic tobacco. Plant cell reports 30:2097-2104

Krasensky J, Jonak C (2012) Drought, salt, and temperature stress-induced metabolic rearrangements and regulatory networks. Journal of experimental botany 63:1593-1608

Kumar K, Rao KP, Sharma P, Sinha AK (2008) Differential regulation of rice mitogen activated protein kinase kinase (MKK) by abiotic stress. Plant physiology and biochemistry : PPB / Societe francaise de physiologie vegetale 46:891-897

Lee SK, Kim BG, Kwon TR, Jeong MJ, Park SR, Lee JW, Byun MO, Kwon HB, Matthews BF, Hong CB, Park SC (2011) Overexpression of the mitogen-activated protein kinase gene OsMAPK33 enhances sensitivity to salt stress in rice (Oryza sativa L.). Journal of biosciences 36:139-151

Li Z, Yue H, Xing D (2012) MAP Kinase 6-mediated activation of vacuolar processing enzyme modulates heat shock-induced programmed cell death in Arabidopsis. The New phytologist 195:8596

Link V, Sinha AK, Vashista P, Hofmann MG, Proels RK, Ehness R, Roitsch T (2002) A heat-activated MAP kinase in tomato: a possible regulator of the heat stress response. FEBS Lett 531:179-183

Mikolajczyk M, Awotunde OS, Muszynska G, Klessig DF, Dobrowolska G (2000) Osmotic stress induces rapid activation of a salicylic acid-induced protein kinase and a homolog of protein kinase ASK1 in tobacco cells. The Plant cell 12:165-178

Mizoguchi T, Irie K, Hirayama T, Hayashida N, Yamaguchi-Shinozaki K, Matsumoto K, Shinozaki K (1996) A gene encoding a mitogen-activated protein kinase kinase kinase is induced simultaneously with genes for a mitogen-activated protein kinase and an $\mathrm{S} 6$ ribosomal protein kinase by touch, cold, and water stress in Arabidopsis thaliana. Proceedings of the National Academy of Sciences of the United States of America 93:765-769

Moustafa K, Lefebvre-De Vos D, Leprince A-S, Savourée A, Laurière C (2008) Analysis of the Arabidopsis Mitogen-Activated Protein Kinase Families: Organ Specificity and Transcriptional Regulation upon Water Stresses. Scholarly Research Exchange 2008:12 pages. doi:10.3814/2008/143656

Nakagami H, Kiegerl S, Hirt H (2004) OMTK1, a novel MAPKKK, channels oxidative stress signaling through direct MAPK interaction. J Biol Chem 279:26959-26966 
Ning J, Li X, Hicks LM, Xiong L (2010) A Raf-Like MAPKKK Gene DSM1 Mediates Drought Resistance through Reactive Oxygen Species Scavenging in Rice. Plant physiology 152:876-890

Pan J, Zhang M, Kong X, Xing X, Liu Y, Zhou Y, Liu Y, Sun L, Li D (2012) ZmMPK17, a novel maize group D MAP kinase gene, is involved in multiple stress responses. Planta 235:661-676

Pastenes C, Horton P (1996) Effect of High Temperature on Photosynthesis in Beans (I. Oxygen Evolution and Chlorophyll Fluorescence). Plant physiology 112:1245-1251

Peng LX, Gu LK, Zheng CC, Li DQ, Shu HR (2006) Expression of MaMAPK gene in seedlings of Malus L. under water stress. Acta Biochim Biophys Sin (Shanghai) 38:281-286

Rengasamy P (2006) World salinization with emphasis on Australia. Journal of experimental botany 57:1017-1023

Roberts EH (1988) Temperature and seed germination. Symposia of the Society for Experimental Biology 42:109-132

Sangwan V, Dhindsa RS (2002) In vivo and in vitro activation of temperature-responsive plant map kinases. FEBS Lett 531:561-564

Sangwan V, Orvar BL, Beyerly J, Hirt H, Dhindsa RS (2002) Opposite changes in membrane fluidity mimic cold and heat stress activation of distinct plant MAP kinase pathways. The Plant journal : for cell and molecular biology 31:629-638

Shi J, Zhang L, An H, Wu C, Guo X (2011) GhMPK16, a novel stress-responsive group D MAPK gene from cotton, is involved in disease resistance and drought sensitivity. BMC molecular biology 12:22

Shou H, Bordallo P, Wang K (2004) Expression of the Nicotiana protein kinase (NPK1) enhanced drought tolerance in transgenic maize. Journal of experimental botany 55:1013-1019

Suri SS, Dhindsa RS (2008) A heat-activated MAP kinase (HAMK) as a mediator of heat shock response in tobacco cells. Plant, cell \& environment 31:218-226

Tanoue T, Adachi M, Moriguchi T, Nishida E (2000) A conserved docking motif in MAP kinases common to substrates, activators and regulators. Nature cell biology 2:110-116

Teige M, Scheikl E, Eulgem T, Doczi R, Ichimura K, Shinozaki K, Dangl JL, Hirt H (2004) The MKK2 pathway mediates cold and salt stress signaling in Arabidopsis. Mol Cell 15:141-152

Vierling E (1991) The role of heat-shock proteins in plants. Annual Review of Plant Physiology and Plant Molecular Biology 42:579-620

Wang J, Ding H, Zhang A, Ma F, Cao J, Jiang M (2010) A novel mitogen-activated protein kinase gene in maize (Zea mays), ZmMPK3, is involved in response to diverse environmental cues. J Integr Plant Biol 52:442-452

Wang M, Zhang Y, Wang J, Wu X, Guo X (2007) A novel MAP kinase gene in cotton (Gossypium hirsutum L.), GhMAPK, is involved in response to diverse environmental stresses. J Biochem Mol Biol 40:325-332 
Wen JQ, Oono K, Imai R (2002) Two novel mitogen-activated protein signaling components, OsMEK1 and OsMAP1, are involved in a moderate low-temperature signaling pathway in rice. Plant physiology 129:1880-1891

Went FW (1953) The Effect of Temperature on Plant Growth. Annual Review of Plant Physiology 4:347-362

Wu T, Kong X-P, Zong X-J, Li D-P, Li D-Q (2011) Expression analysis of five maize MAP kinase genes in response to various abiotic stresses and signal molecules. Molecular biology reports 38:3967-3975

Xie G, Kato H, Imai R (2012) Biochemical identification of the OsMKK6-OsMPK3 signalling pathway for chilling stress tolerance in rice. The Biochemical journal 443:95-102

Xiong L, Ishitani M, Zhu JK (1999) Interaction of osmotic stress, temperature, and abscisic acid in the regulation of gene expression in Arabidopsis. Plant physiology 119:205-212

Xiong L, Yang Y (2003) Disease resistance and abiotic stress tolerance in rice are inversely modulated by an abscisic acid-inducible mitogen-activated protein kinase. The Plant cell 15:745-759

Xu H, Li K, Yang F, Shi Q, Wang X (2010) Overexpression of CsNMAPK in tobacco enhanced seed germination under salt and osmotic stresses. Molecular biology reports 37:3157-3163

Xu J, Chua NH (2012) Dehydration stress activates Arabidopsis MPK6 to signal DCP1 phosphorylation. Embo J 31:1975-1984

Xu J, Li Y, Wang Y, Liu H, Lei L, Yang H, Liu G, Ren D (2008) Activation of MAPK kinase 9 induces ethylene and camalexin biosynthesis and enhances sensitivity to salt stress in Arabidopsis. J Biol Chem 283:26996-27006

Yamazaki T, Kawamura Y, Uemura M (2009) Extracellular freezing-induced mechanical stress and surface area regulation on the plasma membrane in cold-acclimated plant cells. Plant Signal Behav 4:231-233

Yu L, Nie J, Cao C, Jin Y, Yan M, Wang F, Liu J, Xiao Y, Liang Y, Zhang W (2010) Phosphatidic acid mediates salt stress response by regulation of MPK6 in Arabidopsis thaliana. The New phytologist 188:762-773

Zhang L, Xi D, Li S, Gao Z, Zhao S, Shi J, Wu C, Guo X (2011) A cotton group C MAP kinase gene, GhMPK2, positively regulates salt and drought tolerance in tobacco. Plant Mol Biol 77:17-31

\footnotetext{
*Email: khaled.moustafa@frenxiv.org
} 